\title{
Metacognição, ensino-aprendizagem e formação de professores de ciências
}

Metacognition, teaching \& learning and science teacher training

Jesus Cardoso Brabo ${ }^{1}$

\section{Resumo}

São apresentadas algumas razões da importância do desenvolvimento de pesquisas sobre Metacognição e aplicações dos princípios teóricos derivados na elaboração de estratégias de ensino-aprendizagem de alunos e professores de ciências e matemática. Também são apresentados e categorizados os artigos selecionados para esta edição especial.

Palavras chave: metacognição; ensino de ciências; formação de professores.

\section{Abstract}

Some reasons are given for the importance of the development of research on Metacognition and applications of the derived theoretical principles in the elaboration of teaching \& learning science education strategies for students and teachers. The articles selected for this special edition are also presented and categorized.

Keywords: metacognition; science education; teacher training.

\section{Origem e importância do campo de pesquisa}

Apesar de ter se originado no início da década de 1970, a partir de estudos sobre o desenvolvimento dos processos de memorização de crianças (FLAVELL, FRIEDRICHS, HOYT, 1970), cujo desenvolvimento acabou culminando na ideia da existência da chamada metacognição (BROWN, 1978; FLAVEL, 1979), foi só a partir dos anos de 1990 que a quantidade de pesquisas a respeito desse tema começou realmente a se expandir. $\mathrm{Na}$ atualidade, principalmente nos Estados Unidos, Inglaterra, Canadá e Austrália, pesquisas e aplicações vem se multiplicando cada vez mais, ganhando espaço e importância, especialmente na área educacional (ZOHAR e BARZILAI, 2013).

Para tentar traçar um breve panorama dessas pesquisas faremos uma discussão sucinta a respeito da evolução do conceito de metacognição e termos correlatos, os possíveis motivos do crescimento do interesse pelo tema e as possibilidades de pesquisa e aplicações, particularmente relacionadas ao processo de ensino-aprendizagem de ciências e 
matemática e a formação de professores dessas disciplinas. Finalmente, apresentaremos o que este número especial da Amaz RECM sobre metacognição reserva a seus leitores.

Talvez o que tenha chamado mais atenção nas pesquisas pioneiras sobre metacognição tenha sido o fato de que os parâmetros estabelecidos para medir o "conhecimento e a regulação das atividades cognitivas em processos de aprendizagem" se mostraram correlacionados ao bom desempenho acadêmico, ou seja, tais pesquisas concluíram que bons estudantes pareciam possuir habilidades metacognitivas bem desenvolvidas, uma vez que exibiam as qualidades de bons leitores (PARIS e OKA, 1986) e eram bem sucedidos na escola (PINTRICH, DE GROOT, 1990; STERNBERG, 1998).

A partir de então, a medida que os pesquisadores tentavam refinar os componentes e processos (meta)cognitivos, derivados das observações obtidas e hipóteses investigadas, novos termos começaram a proliferar. Metamemória, habilidades metacognitivas, consciência metacognitiva, experiências metacognitivas, conhecimento metacognitivo, habilidades executivas, habilidades de ordem superior, metacomponentes, estratégias de aprendizagem, heurísticas e autoregulação são alguns dos termos que comumente são associados à metacognição. Alguns passaram a referir subcomponentes da metacognição (por exemplo, conhecimento, monitoramento, avaliação e regulação metacognitivos), enquanto outros, apesar de guardar certa relação com a metacognição em si ainda, apresentam certo paralelismo e tem sido foco controvérsias e debates. $O$ conceito de autoregulação (self-regulation of learning), por exemplo, é um destes. Alguns pesquisadores consideram a auto-regulação como um componente subordinado à metacognição (BAKER e BROWN, 1984; FLAVELL, 1985), enquanto outros preferem usá-la com um conceito superordenado à metacognição (ZIMMERMAN, 1995; WINNE, 1996), entre outras variantes. Um dos motivos dessas diferenças de significado se dá em virtude de que os pesquisadores partidários à primeira abordagem citada procuram focar mais atenção em processos cognitivos individuais, enquanto os partidários da segunda assumem uma perspectiva sóciocognitiva, que procura levar em consideração os eventuais processos motivacionais e socioemocionais em jogo. Todavia, a controvérsia segue ativa (DINSMORE, ALEXANDER e LOUGHLIN, 2008).

Outra controvérsia importante refere-se ao debate a respeito da existência ou não da real distinção enttre cognição e metacognição: seriam processos cognitivos de natureza diferentes ou apenas manifestações aparentemente distintas de um processo cognitivo único? Embora considerem pertinente distinguir metacognição e cognição Veenman, Van Hout-Wolters e Afflerbach (2006) admitem que tais processos são altamente interrelacionados, já que ao postular a metacognição sempre requeremos a cognição. De qualquer forma ainda não há consenso sobre o que, de fato, as distingue (THOMAS, 2012).

Apesar dessas e outras controvérsias e certa falta de precisão de sua definição, reflexo de disputas entre as diferentes abordagens de pesquisa dentro da própria área de psicologia, a metacognição só vem ganhando importância nos últimos 30 anos. A inclusão do conceito de conhecimento metacognitivo como uma categoria de destaque da taxonomia revisada de Bloom (KRATHWOHL, 2002; PINTRICH, 2010) é uma demonstração do vigor e da influência dos resultados de pesquisas acumulados ao longo desses anos.

De maneira geral, a pesquisa básica sobre metacognição tem investigado como indivíduos compreendem e regulam sua própria cognição. Ou seja, a forma como uma pessoa se auto-questiona e toma decisões a respeito do que está aprendendo ou quer aprender, pois, teoricamente, são as habilidades de caráter metacognitivo que habilitam o 
indivíduo a perceber melhor suas afinidades e/ou dificuldades com determinado problema e planejar e avaliar a execução das tarefas cognitivas necessárias a esse aprendizado.

Além de problemas de pesquisa básica na área de psicologia cognitiva (o que é? como medir? quais as variações? quais o componentes? etc.), ultimamente muitas pesquisas aplicadas sobre metacognição tem sido feitas em áreas diversas, tais como psiquiatria (LYSAKER et al., 2011), linguística (WENDEN, 1998) e até mesmo ciência da computação (COX, 2005). Especificamente na área educacional, materiais e projetos instrucionais baseados nas teorias da metacognição vem sendo testados em diferentes contextos e níveis de ensino, principalmente em relação com aprendizagem de leitura e escrita (DONNDELINGER, 2005; JOSEPH, 2005) e conteúdos específicos de matemática (CARR, 2010; PAPPAS, GINSBURG e JIANG, 2003; VO et al, 2014) e Ciências (KUHN e PEASE, 2010; CASE E GUNSTONE, 2006; DAVIDOWITZ, ROLLNICK, 2003; ANDERSON, THOMAS, e NASHON, 2009).

\section{Metacognição e ensino e aprendizagem de ciências}

No que diz respeito especificamente ao ensino-aprendizagem de ciências, segundo Thomas (2012), embora grande parte desses estudos declarem que a eventual melhoria da aprendizagem de conceitos científicos dos estudantes esteja entre os objetivos das intervenções didáticas propostas, há um certo consenso que o bom desenvolvimento de habilidades metacognitivas acaba sendo útil para os estudantes aprenderem melhor os mais diferentes temas e habilidades.

Os resultados de intervenções com uso de estratégias metacognitivas vem se mostrando bastante promissores. Pesquisas como as Thomas e Mee (2005) e Thomas (2013), por exemplo, têm demonstrado que com alteração do meio ambiente de aprendizagem e a realização de atividades novas e alternativas, é possível facilitar o desenvolvimento da metacognição dos alunos e melhorar a aprendizagem de ciências dos estudantes. Todavia, essas mesmas pesquisas demonstraram também que para isso ocorrer de forma mais efetiva é necessário que tais atividades ocorram diária e sistematicamente. Em outras palavras, a incorporação de formação metacognitiva em aulas ciências todos os dias parece aumentar as chances de que os alunos fiquem motivados a participar das atividades que lhes são sugeridas.

Desautel (2009) também observa que essa reconfiguração perene do ambiente e clima de sala de aula tem que ocorrer para que estudantes tenham oportunidades de sistematicamente realizar uma reflexão consciente sobre a eficácia dos processos de aprendizagem, atividades e estratégias que empregam ou são convidados a empregar. Por isso o uso de heurísticas (tais como mapas conceituais, gráficos de leitura, diagramas de Venn, fluxogramas de investigação etc.) e quaisquer outros meios de ajudar os alunos a desenvolver e representar seus entendimentos da ciência e seus processos são tão importantes, pois é somente através da reflexão consciente sobre o uso dessas heurísticas e estruturas que metacognição se desenvolve (THOMAS, 2013).

Pesquisas sobre metacognição e ensino-aprendizagem de ciências como as de Thomas e McRobbie (2001) também descobriram que assim como o conhecimento das concepções alternativas dos estudantes é importante para ensinar conceitos e habilidades científicas, também é essencial reconhecer o papel que a "metacognição prévia" dos alunos, incluindo suas crenças sobre a natureza dos processos de aprendizagem, e o que ela desempenha na 
criação e influencia o contexto no qual as intervenções didáticas ocorrem. Uma vez que grande parte dos estudantes, até certo ponto, aprende habilidades metacognitivas de seus pais, colegas e, especialmente, de seus professores.

Essa constatação levou os referidos pesquisadores a propor o chamado Conflito Metacognitivo, análogo ao conflito cognitivo, como método de aprendizagem de habilidades metacognitivas, colocando os alunos em situações nas quais seus quadros conceituais existentes relacionadas com conceitos científicos são desafiados e têm que considerar novas concepções de ciência fenômenos com referência a essas estruturas já existentes (THOMAS e MCROBBIE, 2001).

Os resultados dessas e outras pesquisas tem demonstrado cada vez mais evidências que a metacognição é a chave para atender às agendas múltiplas que caracterizam a educação científica na atualidade. Tais agendas incluem o desenvolvimento do letramento científico dos alunos e sua compreensão da natureza da investigação científica, a natureza dos conceitos científicos e da ciência em si. Por exemplo, para que sejam capazes de realizar um processo de investigação científica, os estudantes devem desenvolver habilidades para realizar conscientemente procedimentos particulares, tanto físicos quanto cognitivos, para monitorar suas respostas aos objetivos da investigação, e, à medida que avançam, devem estar atentos e avaliar os seus progressos, e refletirem sobre os resultados da investigação que estão levando a cabo, com vista a melhorar as suas práticas (THOMAS, 2012). Este tipo de pensamento consciente é a marca metacognitiva individual.

Gunstone (1994) e White (1998), que talvez tenham sido os pioneiros em investigar a relação entre ensino-aprendizagem de ciências e metacognição, observaram que os alunos metacognitivos eram centrais para ambientes de aprendizagem construtivistas, uma vez que esses ambientes pressupõem que os estudantes devem continuamente monitorar novas informações que thes são apresentadas e compará-las com o que eles já sabem de sua aprendizagem anterior. Ou seja, um tipo de reflexão constante, sistemática e consciente que está no coração das teorias de mudança conceitual propostas desde os anos de 1980.

Naturalmente, tal como mostraram as pesquisas de Leat e Lin (2003) e Schreiber (2005), para que os professores adotassem tal abordagem seria imprescindível que: (a) fossem altamente metacognitivos, (b) tivessem uma compreensão completa da natureza e estrutura da área de assunto e material que estão ensinando e que está a ser aprendido, (c) fossem capazes de conversar com os alunos sobre os processos cognitivos e estratégias que podem ser empregadas para melhorar a compreensão conceitual do assunto e (d) fossem capazes de modelar os processos cognitivos e estratégias para os alunos imitarem (ou seja, explicitar para os estudantes suas próprias estratégias cognitivas e metacognitivas). Só assim intervenções que sejam cuidadosamente planejadas e implementadas por professores metacognitivamente preparadas poderão efetivamente tornar o ambiente de sala de aula mais metacognitivamente orientado e assim possibilitar o desenvolvimento e melhoria da metacognição dos estudantes.

Isso implica que o desenvolvimento de habilidades metacognitivas dos alunos exige que os professores de ciências sejam eles próprios metacognitivos e capazes de se comunicar com os alunos sobre os benefícios de maneiras específicas de pensar sobre a aprendizagem da ciência e como este pode ser melhor facilitado (WILSON e BAI, 2010).

Pesquisas desenvolvidas por Zohar $(1999,2004)$ tem destacado a importância do conhecimento metacognitivo de professores e a dificuldade que eles têm em mudar da instrução tradicional para uma que incida sobre o ensino do pensamento de ordem 
superior. Ela também observou a dificuldade que os professores têm em articular seus padrões de pensamento durante a resolução de problemas e concluiu que conhecimento declarativo metacognitivo dos professores é essencial para o ensino do pensamento de ordem superior.

Infelizmente toda a importância dada ao desenvolvimento de habilidades metacognitivas dos estudantes, e de suas próprias, está longe de ser seriamente considerada pela maioria dos professores ou formadores de professores (THOMAS, 2012; VEENMAN, VAN HOUT-WOLTERS e AFFLERBACH, 2006). Mesmo em países como os Estados Unidos, Austrália e Inglaterra, onde a pesquisa e desenvolvimento de projetos de ensino metacognitivamente fundamentadas estão em curso desde a década de 1980 (FEUERSTEIN, 1985; MITCHELL e MITCHELL, 2008), aplicações das pesquisas sobre metacognição ainda estão muito distantes das escolas (PERRY, LUNDIE e GOLDER, 2018).

No Brasil o interesse de pesquisadores da área educacional pelo tema ainda está engatinhando. Isso pode ser constatado na pequena quantidade de resultados que retornam quando se busca pelo termo metacognição e correlatos, em português, nas principais bases de dados nacionais. Apesar de já na década de 1990 terem sido realizados alguns estudos de pesquisadores brasileiros sobre metacognição, a grande maioria relacionada a leitura e interpretação de textos, só muito recentemente foram defendidas as primeiras teses de doutorado de universidades brasileiras especificamente focadas na investigação do papel das habilidades metacognitivas no processo de ensino-aprendizagem de Física (ROSA, 2011) e Química (LOCATELLI, 2016).

Esse número especial da Amaz RECM tem justamente a intenção de impulsionar a realização de pesquisas sobre esse tema, reunindo trabalhos de pesquisadores brasileiros que desenvolvem pesquisas relacionadas à metacognição. Tal esforço conseguiu reunir doze artigos que ilustram o que se tem feito atualmente no país.

Basicamente os trabalhos selecionados para esta edição podem ser classificados em quatro grupos distintos. O primeiro grupo refere-se a artigos de revisão, representado pelo manuscrito de Cleophas e Francisco (2018) que faz uma revisão sistemática de artigos sobre metacognição - publicados em português ou espanhol, disponíveis em banco de dados de acesso livre; mostrando o quanto, no Brasil e na América Latina, o tema é ainda é pouco pesquisado na área de ensino de ciências em relação outras temáticas.

O segundo grupo de artigos que compõem este número especial, representados pelos trabalhos de Medeiros, Silva e Locatelli (2018), Ferreira, Bernadi, Silveira e Freitas (2018), Tavares, Muller e Fernandes (2018) e Locatelli e Alves (2018), investigaram o impacto da utilização de diferentes estratégias de ensino no desenvolvimento de habilidades metacognitivas de estudantes.

Os artigos de Darroz, Travisan e Rosa (2018), Lucas e Pereira (2018), Lima Silva e Noronha (2018), Rosa, Ribeiro e Rosa (2018) e Maffei e Silva (2018), por outro lado realizaram sondagens de habilidades metacognitivas apresentadas por estudantes de diferentes graus de escolaridade, procurando correlações entre essas supostas habilidades e o desempenho escolar.

Finalmente os artigos de Boni e Laburú (2018) e Rocha e Malheiro (2018) apresentam uma discussão teórica em torno de propostas de instrumentos de análise que, eventualmente, poderão ser utilizados para analisar habilidades cognitivas e metacognitivas em situações didáticas específicas. 
Esperamos que os artigos publicados nesta edição além de servir como fonte de referências sobre o tema, possam ajudar também formadores de professores a formular e/ou aperfeiçoar atividades educativas de natureza metacognitiva. Bem como estimulá-los a desenvolver pesquisas nessa linha. Tal como foi discutido nesse breve editorial, temos várias razões para acreditar que estudo da teoria e a prática de uso de estratégias metacognitivas por parte de professores de ciências e matemática poderá fazê-los tomar consciência da importância das vantagens de usar essas habilidades em suas vidas profissionais e acadêmicas e ensiná-las aos seus alunos, crianças ou adultos.

Finalmente, não poderíamos deixar de agradecer aos autores e aos avaliadores ad hoc da revista, sem os quais o lançamento deste número e de outros não seria possível. Dedico um especial agradecimento a Profa. Dra. Solange Locattelli da UFABC pela coordenação geral da edição deste número especial da Amaz RECM.

\section{Referências}

ANDERSON, D; THOMAS, G; NASHON, S. Social barriers to meaningful engagement in biology field trip group work.Science Education, v. 93, n. 3, p.511-534, 2009.

BAKER, L; BROWN, A. Metacognitive skills and reading. In: PEARSON, P. D;

BARR, R; KAMIL, M; MOSENTHAL, P. (Eds.). Handbook of Reading Research. New York: Longman, 1984.p.353-394.

BONI, Keila Tatiana; LABURÚ , Carlos Eduardo. Conceitualização e metacognição em Ciências e Matemática: pressupostos teóricos de um instrumento analítico. Amazônia: Revista de Educação em Ciências e Matemáticas, v. 14, n. 29, p. 177-192, 2018.

BROWN, A. Knowing when, where, and how to remember: a problem of metacognition. In: GLASER, R. (Ed.). Advances in instructional psychology, v.2. Hillsdale, NJ: Erlbaum, 1978. p.77-165.

CARR, M. (2010). The importance of metacognition for conceptual change and strategy use in mathematics.In WATERS, H. S; SCHNEIDER, W. (Eds.).Metacognition, strategy use, and instruction. New York: The Guilford Press, 2010, p.176-199.

CASE, J.; GUNSTONE, R. Metacognitive development: a view beyond cognition. Research in science education, v.36, n.1-2, p.51-67, 2006.

CLEOPHAS, Maria das Graças; FRANCISCO, Welington. Metacognição e o ensino e aprendizagem das ciências: uma revisão sistemática da literatura (RSL). Amazônia: Revista de Educação em Ciências e Matemáticas, v. 14, n. 29, p. 10-26, 2018.

COX, Michael T. Field review: Metacognition in computation: a selected research review. Artificial intelligence, v.169, n. 2, p.104-141, 2005.

DARROZ, Luiz Marcelo; TRAVISAN, Thais Lorençato; ROSA, Cleci Teresinha Werner da. Estratégias de aprendizagem: caminhos para o sucesso escolar. Amazônia: Revista de Educação em Ciências e Matemáticas, v. 14, n. 29, p. 93-109, 2018.

DAVIDOWITZ, B; ROLLNICK, M. Enabling metacognition in the laboratory: A case study of four second year university chemistry students. Research in Science Education, v.33, n.1, p.43-69, 2003.

DESAUTEL, D. Becoming a Thinking Thinker: Metacognition, Self-Reflection, and Classroom Practice. Teachers College Record, v.111, n.8, p.1997-2020, 2009. 
DINSMORE, D; ALEXANDER, P; LOUGHLIN, S. Focusing the conceptual lens on metacognition, self-regulation, and self-regulated learning. Educational Psychology Review, v.20, n.4, p.391-409, 2008.

DONNDELINGER, S. J. Integrating comprehension and metacognitive reading strategies. In: ISRAEL, S. et al. Metacognition in literacy learning: theory assessment, instruction, and professional development. Mahwah, N.J.: Lawrence Erlbaum, 2005. p.41-59.

FERREIRA, Sabrina Antunes; BERNARDI, Geovane; SILVEIRA, Maira dos Santos; LEONARDI, Ariadne de Freitas; GOLDSCHMIDT, Andréa Inês. Atividades metacognitivas como facilitadoras na aprendizagem sobre seres vivos nos anos iniciais. Amazônia: Revista de Educação em Ciências e Matemáticas, v. 14, n. 29, p. 43-62, 2018.

FEUERSTEIN, R. Instrumental enrichment: an intervention program for cognitive modifiability. Baltimore: University Park Press, 1985.

FLAVELL, J. Cognitive development. 2a. ed. Englewood Cliffs, NJ: Prentice Hall, 1985. FLAVELL, J. Metacognition and cognitive monitoring: a new area of cognitive-developmental inquiry. American psychologist, v.34, n.10, p.906, 1979.

FLAVELL, J; FRIEDRICHS, A; HOYT, J. Developmental changes in memorization processes.Cognitive psychology, v.1, n.4, p.324-340, 1970.

GUNSTONE, R. The importance of specific science content in the enhancement of metacognition. In: FENSHAM, P; GUNSTONE, R. e WHITE, R. (Eds.). The content of science: a constructivist approach to its learning and teaching. London: Falmer Press, 1994. p.131-146.

JOSEPH, L. The role of self-monitoring in literacy learning.In: ISRAEL, S. et al. Metacognition in literacy learning: theory assessment, instruction, and professional development. Mahwah, N.J: Lawrence Erlbaum, 2005. p.199-214.

KRATHWOHL, David R. A revision of Bloom's taxonomy: an overview. Theory into practice, v.41, n.4, p.212-218, 2002.

KUHN, D.; PEASE, M. The dual components of developing strategy use: production and inhibition. In: WATERS, H. S.; SCHNEIDER, W. (Eds.). Metacognition, strategy use, and instruction. New York: Guilford Press, 2010. p.135-159.

LEAT, D; LIN, M. Developing a pedagogy of metacognition and transfer: some signposts for the generation and use of knowledge and the creation of research partnerships. British Educational Research Journal, v.29, n.3, p.383-414, 2003.

LIMA, Pablo Jovellano dos Santos; SILVA, Marcia Gorette Lima da; NORONHA, Claudianny Amorim. Estratégias metacognitivas na resolução de problemas verbais de matemática no ensino fundamental. Amazônia: Revista de Educação em Ciências e Matemáticas, v. 14, n. 29, p. 125-142, 2018.

LOCATELLI, Solange Wagner. Relação existente entre metavisualização e as representações simbólica e submicro na elaboração de atividade em química. Tese de Doutorado. Universidade de São Paulo, São Paulo, Brasil, 2016.

LOCATELLI, Solange Wagner; ALVES, Natália Cristina Barbosa. Aproximações entre o monitoramento metacognitivo e a elaboração de portfólio em uma disciplina de Química Geral. Amazônia: Revista de Educação em Ciências e Matemáticas, v. 14, n. 29, p. 79-92, 2018.

LUCAS, Atos Edwin Pereira da Silva; PEREIRA, Marta Maximo. A reflexão dos estudantes sobre a tarefa de elaborar questões de Física: um olhar ao longo do tempo. Amazônia: Revista de Educação em Ciências e Matemáticas, v. 14, n. 29, p. 110-124, 2018. 
LYSAKER, Paul H. et al. Addressing metacognitive capacity for self reflection in the psychotherapy for schizophrenia: a conceptual model of the key tasks and processes.Psychology and Psychotherapy: Theory, Research and Practice, v.84, n.1, p.58-69, 2011.

MAFFEI, Letícia Queiroz; SILVA, João Alberto da. O que se mostra quando pedagogas em formação escrevem sobre suas experiências com a matemática? Amazônia: Revista de Educação em Ciências e Matemáticas, v. 14, n. 29, p. 161-176, 2018.

MEDEIROS, Edilene Ferreira de; SILVA, Marcia Gorette Lima da; LOCATELLI, Solange Wagner. A argumentação e o potencial metacognitivo de uma atividade experimental baseada na POA (Previsão-Observação-Argumentação). Amazônia: Revista de Educação em Ciências e Matemáticas, v. 14, n. 29, p.27-42, 2018.

MITCHELL, Ian; MITCHELL, Judie. The project for enhancing effective learning (PEEL): 22 years of praxis. In: SAMARAS, A; FREESE, A; KOSNIK, C; BECK, C. (Eds.). Learning communities in practice. Dordrecht: Springer, 2008. v.4, p.7-18.

PAPPAS, S; GINSBURG, H.; JIANG, M. SES differences in young children's metacognition in the context of mathematical problem solving. Cognitive Development, v. 18, n. 3, p. 431-450, 2003.

PARIS, S; OKA, E. Children's reading strategies, metacognition, and motivation. Developmental review, v.6, n.1, p.25-56, 1986.

PERRY, J; LUNDIE, D; GOLDER, G. Metacognition in schools: what does the literature suggest about the effectiveness of teaching metacognition in schools? Educational Review, p.1-18, 2018.

PINTRICH, P. R. The Role of Metacognitive Knowledge in Learning, Teaching, and Assessing. Theory Into Practice, v.41, n.4, p.219-225, 2010.

PINTRICH, P; DE GROOT, E. Motivational and self-regulated learning components of classroom academic performance.Journal of Educational Psychology, v.82, n.1, p.33, 1990.

ROCHA, Carlos José Trindade da; MALHEIRO, João Manoel da Silva. Interações dialógicas na experimentação investigativa em um clube de ciências: proposição de instrumento de análise metacognitivo. Amazônia: Revista de Educação em Ciências e Matemáticas, v. 14, n. 29, p.193-207, 2018.

ROSA, Cleci T. Werner da.A metacognição e as atividades experimentais no ensino de Física. Tese de Doutorado. Universidade Federal de Santa Catarina, Florianópolis, Brasil, 2011.

ROSA, Cleci Teresinha Werner da; RIBEIRO, Cassia de Andrade Gomes; ROSA, Alvaro Becker da. Habilidades metacognitivas envolvidas na resolução de problemas em Física: Investigando estudantes com expertise. Amazônia: Revista de Educação em Ciências e Matemáticas, v. 14, n. 29, p. 143-160, 2018.

SCHREIBER, F. Metacognition and self-regulation in literacy. In: ISRAEL, S; BLOCK, C; BAUSERMAN, K; KINNUCAN-WELSCH, K. (Eds.). Metacognition in literacy learning: theory, assessment, instruction, and professional development. Mahwah, NJ: Lawrence Erlbaum, 2005. p.215-239.

SCHUNK, Dale H. Metacognition, self-regulation, and self-regulated learning: Research recommendations. Educational psychology review, v.20, n.4, p.463-467, 2008.

STERNBERG, Robert J. Metacognition, abilities, and developing expertise: what makes an expert student? Instructional Science, v.26, n.1-2, p.127-140, 1998.

TAVARES, Laís Conceição; MÜLLER, Regina Celi Sarkis; FERNANDES, Adriano Caldeira. O uso de mapas conceituais como ferramenta metacognitiva no ensino de Química. Amazônia: Revista de Educação em Ciências e Matemáticas, v. 14, n. 29, p.63-78, 2018. 
THOMAS, G. Changing the metacognitive orientation of a classroom environment to stimulate metacognitive reflection regarding the nature of physics learning. International Journal of Science Education, v. 35, n. 7, p. 1183-1207, 2013.

THOMAS, G. Metacognition in science education: past, present, and future considerations. In: FRASER, B; TOBIN, K; MCROBBIE, C. (Eds.). Second International Handbook of Science Education. 2. ed. [S.I.]: Springer, 2012. p.131-144.

THOMAS, G; MCROBBIE, C.J. Using a metaphor for learning to improve students' metacognition in the chemistry classroom.Journal of Research in Science Teaching, v.38, n.2, p. 222-259, 2001.

THOMAS, G; MEE, D. Changing the learning environment to enhance students' metacognition in Hong Kong primary school classrooms.Learning Environments Research, v.8, n.3, p.221-243, 2005.

VEENMAN, M; VAN HOUT-WOLTERS, B; AFFLERBACH, P. Metacognition and learning: Conceptual and methodological considerations. Metacognition and learning, v.1, n.1, p.3-14, 2006.

$\vee O, V$. et al. Young children bet on their numerical skills: metacognition in the numerical domain. Psychological Science, v.25, n.9, p.1712-1721, 2014.

WENDEN, A. Metacognitive knowledge and language learning1. Applied linguistics, v.19, n.4, p.515537, 1998.

WHITE, R. Decisions and problems in research on metacognition. In: FRASER, B; TOBIN, K. (Eds.).

International Handbook of Science Education. Dordrecht: Kluwer, 1998. p.1207-1213.

WILSON, N; BAl, H.The relationships and impact of teachers' metacognitive knowledge and pedagogical understandings of metacognition. Metacognition and Learning, v.5, n.3, p.269-288, 2010.

WINNE, P. A metacognitive view of individual differences in self-regulated learning.Learning and individual differences, v.8, n.4, p.327-353, 1996.

YOUNG, A; FRY, J. Metacognitive awareness and academic achievement in college students. Journal of the Scholarship of Teaching and Learning, v.8, n.2, p.1-10, 2012.

ZIMMERMAN, Barry J. Self-regulation involves more than metacognition: a social cognitive perspective. Educational Psychologist, v.30, n.4, p.217-221, 1995.

ZOHAR A; BARZILAI, S. A review of research on metacognition in science education: current and future directions. Studies in Science Education, v.49, n. 2, p.121-169, 2013.

ZOHAR, A. Higher order thinking in science classrooms: students' learning and teachers' professional development. Dordrecht: Springer, 2004.

ZOHAR, A. Teachers' metacognitive knowledge and the instruction of higher order thinking. Teaching and teacher Education, v.15, n.4, p.413-429, 1999. 